\title{
Design and Implementation of an Improved Apriori Data Mining Algorithm
}

\author{
Meilin Zeng ${ }^{1, \mathrm{a}^{*}}$,Qiangqiang Xiong ${ }^{2, \mathrm{~b}}$ and $\mathrm{Ke} \mathrm{Li}^{3, \mathrm{c}}$ \\ 1Jiangxi Vocational Technical College of Industry Trade Nanchang 330038 \\ ${ }^{2}$ Nanchang institute of technology Nanchang 330013 \\ * The Corresponding Author
}

Keyword: Data Mining; Association Rules; Frequent Itemsets; Apriori Algorithm

\begin{abstract}
The need to scan the database D many times when the Apriori algorithm is applied to a large database causes the I/O load overhead of the disk to increase. An improved Apriori algorithm is designed. After scanning the original database D for the first time, it generates a candidate transaction database $A_{k}$. In the process of generating frequent itemsets, the candidate transaction database $A_{k}$ is scanned each time. Experiments show that if the $\mathrm{K}$ value is very large, the number of $A_{k}$ will be much less than that of the original database, which will solve the problem of $\mathrm{I} / \mathrm{O}$ overload and reduce operation time, so as to achieve the purpose of optimizing Apriori algorithm.
\end{abstract}

\section{Introduction}

Association rules mining is an important research area in the field of data mining, and aims to mine interesting associations in transactional databases. Apriori algorithm is proposed by Agrawal et al. However, Apriori algorithm has two bottlenecks: a). Scanning the transaction database multiple times requires a lot of I/O load; b). Generating a large set of candidates, this is a challenge to run time and main memory space.

Aiming at the disadvantages of Apriori algorithm, such as low efficiency of generating candidate itemsets and frequent scanning of database, some improved algorithms are proposed. Some papers propose to reduce the number of scan candidate sets by first finding the maximal frequent K-sets and then finding the remaining frequent-sets. However, these methods are relatively complicated. To this end, this paper presents a simple and easy method. After the first scan of the transaction database $\mathrm{D}$, the algorithm not only generates a database of candidate transactions, but only needs to scan candidate transactions in the process of generating frequent itemsets Database, instead of repeatedly scanning the original database $\mathrm{D}$, so as to better solve the first bottleneck, to improve the problem of excessive I/O load, reduce the computing time and achieve the goal of optimizing the Apriori algorithm.

\section{Analysis of Apriori Algorithm}

Implementation Principle of Apriori Algorithm. As a classic data mining algorithm, Apriori algorithm occupies an important position. The basic idea is as follows:

a). By scanning all the data itemsets in the transaction database, a candidate 1 -itemset is obtained, denoted $C_{1}$, and the frequency of occurrence of the corresponding data item is counted. According to the pre-defined minimum support, selected frequent 1-itemsets from $C_{1}$ are screened as $L_{1}$.

b). By frequent self-connection of 1-itemset $L_{1}$, a candidate 2-itemset $C_{2}$ is obtained, and then the transaction database is scanned again to calculate the corresponding frequency so as to be compared with the minimum support to obtain $L_{2}$.

c). Repeat the above steps until there are no longer frequent $\mathrm{K}$-itemsets that meet the requirements. 
It can be seen from the above that Apriori algorithm uses the iterative method of layer-by-layer search to generate high-dimensional frequent items through low-dimensional frequent itemsets. Each mining a frequent item set $L_{k}$, you need to scan the entire transaction database. In the process, we also generate a large number of candidate sets, making Apriori algorithm less efficient.

Key Steps of Apriori. The Apriori algorithm is an iterative way to find the $L_{k}$ by the $L_{k-1}$, so the key steps are: connection, pruning.

a). Connection Step: In order to obtain the $L_{k}, L_{k-1}$ needs to be connected with itself to generate a candidate $-\mathrm{K}$ item set, and the premise of performing is that the elements of th e $L_{k-1}$ are connectable.

b). Prune Steps: $C_{k}$ members may or may not be frequent, but all frequent K-itemsets ar e included in the $C_{k}$. The $C_{k}$ is determined by scanning the database to determine the freq uency of each candidate in the $L_{k}$.

Inefficiency reasons of Apriori algorithm. Apriori algorithm can get frequent itemsets more efficiently, but there are some shortcomings.

a). Whenever mining $\mathrm{K}$ frequent itemsets, the transaction database must be scanned multi ple times to get the corresponding support count, which will inevitably lead to long time c onsumption.

b). A lot of candidate sets will be generated. When the $L_{k-1}$ gets a $L_{k}$ by self-connectio $\mathrm{n}$, a large number of candidate sets may be generated, especially $C_{2}$.

\section{The Thought and Realization of Improved Apriori Algorithm}

The above analysis shows that the main reason for the inefficiency of Apriori algorithm is that the transaction database D needs to be repeatedly scanned. If the size of the transaction database D is small, the problem is not obvious. But obviously the vast majority of databases that need data mining are data warehouses that have massive amounts of data. Therefore, this problem will become cannot be ignored. The Apriori algorithm is also optimized for the sake of streamlining the transaction database $\mathrm{D}$ being scanned every time a frequent item set is generated through a candidate set.

The basic idea of the optimization algorithm is to generate a candidate transaction database $A_{k}$ after the first scan of the original database D. In the process of generating frequent itemsets, each time the original transaction database is no longer scanned, the candidate transaction database $A_{k}$ is scanned, so as to achieve the purpose of improving the efficiency of the algorithm. The elements in candidate transaction database $A_{k}$ are denoted as $\left\langle T I D,\left\{X_{k}\right\}\right\rangle$, where $X_{k}$ represents a frequent set of $\mathrm{X}$-items that may be present. $A_{k}$ specifies the following: $A_{k}=\mathrm{D}$ when $\mathrm{k}=1$. When $\mathrm{K}>1$, the set $A_{k}=$ transaction $\mathrm{S},<s . T I D,\left\{C \in A_{k} \mid C \subset s\right\}>$.

The pseudo-code for generating frequent itemsets $L_{k}$ in the improved Apriori algorithm is as follows:

Enter: Database D, the minimum support min-sup

Output: frequent itemset $\mathrm{L}$ in database $\mathrm{D}$.

$A_{1}:=$ Database D;

$L_{1}:=\left\{C \in A_{1} \mid C\right.$.count $\left.\geq \min -\sup \right\} ; / /$ min-sup stands for the minimum support

$\mathrm{K}:=2$;

while $\left(L_{k}-1\right)$ do

$\left\{C_{k}=\right.$ Apriori_hx $\left(L_{k-1}\right)$; // Apriori_hx () is a candidate project set generation function $C_{k}=\mathrm{F}$; for all $S \in A_{k-1}$ do 
$\left\{C_{s}=\left\{C \in C_{k} \mid c-c[k] \in\right.\right.$ s.set-itemsets $\Lambda c-c[k-1] \in$ s.set-itemsets $\} \quad$ if $\left(C_{s} \neq \mathrm{F}\right)$ then $A_{k}+=<s . T I D, C_{s}>$;

/\}

$L_{k}:=\left\{C \in C_{k} \mid C . c o u n t \geq \min -\sup \right\} ;$

/\}

D_items: $=U_{k} L_{k} ; / / \mathrm{D} \_$items represents all the big item sets

\section{Application of Improved Apriori Algorithm}

There is a database $D$, things $|D|=4$, the minimum support count min-sup=2, the Apriori algorithm and the Apriori Apriori algorithm Apriori algorithm described in detail below, of which Fig. 1 is Apriori algorithm operation graph, Fig. 2 is Apriori algorithm operation process diagram.

\begin{tabular}{|c|c|c|c|c|c|c|c|}
\hline \multicolumn{2}{|c|}{ Database D } & \multirow{7}{*}{ ScanD } & \multicolumn{2}{|c|}{$A_{1}$} & \multirow{7}{*}{ Crude } & \multicolumn{2}{|c|}{$L_{1}$} \\
\hline Transaction & Items & & item-sets & Support & & item-sets & Support \\
\hline$K_{1}$ & $\begin{array}{l}B_{1}, B_{2}, \\
B_{3}, B_{4}\end{array}$ & & $\left\{B_{1}\right\}$ & 3 & & $\left\{B_{1}\right\}$ & 3 \\
\hline$K_{2}$ & $B_{1}, B_{3}$ & & $\left\{B_{2}\right\}$ & 2 & & $\left\{B_{2}\right\}$ & 2 \\
\hline$K_{3}$ & $\begin{array}{l}B_{1}, B_{3} \\
B_{4}\end{array}$ & & $\left\{B_{3}\right\}$ & 4 & & $\left\{B_{3}\right\}$ & 4 \\
\hline \multirow{2}{*}{$K_{4}$} & \multirow{2}{*}{$\begin{array}{l}B_{2}, B_{3}, \\
B_{5}\end{array}$} & & $\left\{B_{4}\right\}$ & 2 & & \multirow{2}{*}{$B_{4}$} & \multirow{2}{*}{2} \\
\hline & & & $\left\{B_{5}\right\}$ & 4 & & & \\
\hline
\end{tabular}

Figure 1. Finite Frequent itemsets $L_{1}$ is generated by candidate frequent itemsets $C_{1}$

\begin{tabular}{|c|c|c|c|c|c|c|}
\hline$C_{2}$ & \multirow{8}{*}{ Scan D } & \multicolumn{2}{|c|}{$A_{2}$} & & \multicolumn{2}{|c|}{$L_{2}$} \\
\hline item-sets & & item-sets & Support & & item-sets & Support \\
\hline$\left\{B_{1}, B_{2}\right\}$ & & $\left\{B_{1}, B_{2}\right\}$ & 1 & & $\left\{B_{1}, B_{3}\right\}$ & 3 \\
\hline$\left\{B_{1}, B_{3}\right\}$ & & $\left\{B_{1}, B_{3}\right\}$ & 3 & \multirow{5}{*}{ Crude } & $\left\{B_{1}, B_{4}\right\}$ & 2 \\
\hline$\left\{B_{1}, B_{4}\right\}$ & & $\left\{B_{1}, B_{4}\right\}$ & 2 & & $\left\{B_{2}, B_{3}\right\}$ & 2 \\
\hline$\left\{B_{2}, B_{3}\right\}$ & & $\left\{B_{2}, B_{3}\right\}$ & 2 & & \multirow{3}{*}{$B_{3}, B_{4}$} & \multirow{3}{*}{2} \\
\hline$\left\{B_{2}, B_{4}\right\}$ & & $\left\{B_{2}, B_{4}\right\}$ & 1 & & & \\
\hline$\left\{B_{3}, B_{4}\right\}$ & & $\left\{B_{3}, B_{4}\right\}$ & 1 & & & \\
\hline
\end{tabular}

Figure 2. Finite Frequent itemsets $C_{2}$ generate frequent itemsets $L_{2}$ 


\begin{tabular}{|c|c|c|c|c|c|c|}
\hline$C_{3}$ & \multirow{3}{*}{ ScanD } & \multicolumn{2}{|c|}{$A_{3}$} & \multirow{3}{*}{ Crude } & \multicolumn{2}{|c|}{$L_{3}$} \\
\hline item-sets & & Transaction & $\begin{array}{l}\text { item-sets } \\
\text { gather }\end{array}$ & & item-sets & Support \\
\hline$\left\{B_{1}, B_{3}, B_{4}\right\}$ & & $\left\{B_{1}, B_{3}, B_{4}\right\}$ & 2 & & $\left\{B_{1}, B_{3}, B_{4}\right\}$ & 2 \\
\hline
\end{tabular}

Figure 3. Finite The frequent itemsets $L_{3}$ is generated by the candidate frequent itemsets $C_{3}$

\begin{tabular}{|c|c|c|c|c|c|c|c|}
\hline \multicolumn{2}{|c|}{ Database D } & \multirow{6}{*}{$\begin{array}{c}\text { Scan } \\
\text { D }\end{array}$} & & $A_{1}$ & \multirow{6}{*}{$\begin{array}{c}\text { Scan } \\
A_{1}\end{array}$} & \multicolumn{2}{|c|}{$L_{1}$} \\
\hline Transaction & Items & & Transaction & item-sets gather & & $\begin{array}{l}\text { item- } \\
\text { sets }\end{array}$ & Support \\
\hline$K_{1}$ & $\begin{array}{l}B_{1}, B_{2}, \\
B_{3}, B_{4}\end{array}$ & & $\left\{K_{1}\right\}$ & $\left\{B_{1}\right\},\left\{B_{2}\right\},\left\{B_{3}\right\},\left\{B_{4}\right\}$ & & $\left\{B_{1}\right\}$ & 3 \\
\hline$K_{2}$ & $B_{1}, B_{3}$ & & $\left\{K_{2}\right\}$ & $\left\{B_{1}\right\},\left\{B_{3}\right\}$ & & $\left\{B_{2}\right\}$ & 2 \\
\hline$K_{3}$ & $\begin{array}{l}B_{1}, B_{3}, \\
B_{4}\end{array}$ & & $\left\{K_{3}\right\}$ & $\left\{B_{1}\right\},\left\{B_{3}\right\},\left\{B_{4}\right\}$ & & $\left\{B_{3}\right\}$ & 4 \\
\hline$K_{4}$ & $\begin{array}{l}B_{2}, B_{3}, \\
B_{5}\end{array}$ & & $\left\{K_{4}\right\}$ & $\left\{B_{2}\right\},\left\{B_{3}\right\},\left\{B_{4}\right\}$ & & $\left\{B_{4}\right\}$ & 2 \\
\hline
\end{tabular}

Figure 4. Finite The frequent item set $L_{1}$ is generated by the candidate transaction database $A_{1}$

\begin{tabular}{|c|c|c|c|c|c|c|}
\hline$C_{2}$ & \multirow{8}{*}{$\begin{array}{c}\text { Scan } \\
\text { D }\end{array}$} & & $A_{2}$ & \multirow{8}{*}{$\begin{array}{c}\text { Scan } \\
A_{2}\end{array}$} & \multicolumn{2}{|c|}{$L_{2}$} \\
\hline item-sets & & Transaction & item-sets gather & & $\begin{array}{c}\text { item- } \\
\text { sets }\end{array}$ & Support \\
\hline$\left\{B_{1}, B_{2}\right\}$ & & $\left\{K_{1}\right\}$ & $\left\{\begin{array}{l}\left\{B_{1}, B_{2}\right\},\left\{B_{1}, B_{3}\right\},\left\{B_{1}, B_{4}\right\} \\
\left\{B_{2}, B_{3}\right\},\left\{B_{2}, B_{4}\right\},\left\{B_{3}, B_{4}\right\}\end{array}\right\}$ & & $\left\{B_{1}, B_{3}\right\}$ & 3 \\
\hline$\left\{B_{1}, B_{3}\right\}$ & & $\left\{K_{2}\right\}$ & $\left\{B_{1}, B_{3}\right\}$ & & $\left\{B_{1}, B_{4}\right\}$ & 2 \\
\hline$\left\{B_{1}, B_{4}\right\}$ & & $\left\{K_{3}\right\}$ & $\left\{B_{1}, B_{3}\right\},\left\{B_{1}, B_{4}\right\},\left\{B_{3}, B_{4}\right\}$ & & $\left\{B_{2}, B_{3}\right\}$ & 2 \\
\hline$\left\{B_{2}, B_{3}\right\}$ & & \multirow{3}{*}{$K_{4}$} & \multirow{3}{*}{$B_{2}, B_{3}$} & & \multirow{3}{*}{$B_{3}, B_{4}$} & \multirow{3}{*}{2} \\
\hline$\left\{B_{2}, B_{4}\right\}$ & & & & & & \\
\hline$\left\{B_{3}, B_{4}\right\}$ & & & & & & \\
\hline
\end{tabular}

Figure 5. Finite The frequent itemsets $L_{2}$ is generated by the candidate transaction database $A_{2}$

\begin{tabular}{|c|c|c|c|c|c|c|}
\hline$C_{3}$ & \multirow{4}{*}{ Scan D } & \multicolumn{2}{|c|}{$A_{3}$} & \multirow{4}{*}{$\begin{array}{c}\text { Scan } \\
A_{3}\end{array}$} & \multicolumn{2}{|c|}{$L_{3}$} \\
\hline Transaction & & Transaction & $\begin{array}{c}\text { item-sets } \\
\text { gather }\end{array}$ & & item-sets & Support \\
\hline \multirow{2}{*}{$B_{1}, B_{2}, B_{3}$} & & $K_{1}$ & $\left\{B_{1}, B_{2}, B_{3}\right\}$ & & $\left\{B_{1}, B_{2}, B_{3}\right\}$ & \multirow{2}{*}{2} \\
\hline & & $K_{3}$ & $\left\{B_{1}, B_{2}, B_{3}\right\}$ & & & \\
\hline
\end{tabular}

Figure 6. Finite Frequent itemsets $L_{3}$ is generated by the candidate transaction database $A_{3}$

Although both the Apriori algorithm and the improved Apriori algorithm generate the frequent itemsets in three steps, the Apriori algorithm scans the original database three times, whereas the improved Apriori algorithm only scans the original database in the first step, The second and third step scan are the introduction of the $A_{k}$ that is, the candidate transaction database, we can see that if the value of $\mathrm{K}$ is very large, the number of $A_{k}$ will be much smaller than the original database, this will solve the I/O load is too large Reduce the computation time and achieve the goal of optimizing Apriori algorithm. 


\section{Experimental Results}

In order to more intuitively verify that the improved algorithm priori algorithm compares the superiority of Apriori in performance, performance testing experiments are conducted on the same computer. The software and hardware configuration of the computer is as follows: Hardware Configuration: Processor: Intel (R) Core (TM) i3-3110M CPU @ 2.40GHz, memory: 2.0GB.Software Platform: Windows 7 Ultimate, Microsoft SQL Server 2005, Microsoft Visual Basic 6.0

The database used in this experiment is a teacher evaluation database of Jiangxi Institute of Industry and Trade. Random sampling of teachers of different grades and different professions in the whole hospital is carried out and contains 600 sets of data. The performance test of the two algorithms adopts fixed minimum support and Transform the method of minimum support for testing.

Fixed Minimum Support. Set the minimum support min-sup $=20 \%$, candidates frequent k-item set respectively $C_{1}, C_{2} \ldots C_{k}, C_{k+1} \ldots$, Apriori, improved Apriori two algorithms to generate candidate frequent k- Shown:

Table 1 The time required for both algorithms to generate candidate frequent k-itemsets $(\mathrm{ms})$

\begin{tabular}{|c|c|c|c|c|c|}
\hline Candidateitem-sets & $C_{1}$ & $C_{2}$ & $C_{3}$ & $C_{4}$ & $C_{5}$ \\
\hline Apriori Algorithm & 163 & 176 & 245 & 192 & 141 \\
\hline $\begin{array}{c}\text { Improved Apriori } \\
\text { Algorithm }\end{array}$ & 164 & 92 & 129 & 87 & 0 \\
\hline
\end{tabular}

It can be seen from the figure above that in the case of fixed minimum support, except for the time required to generate candidate frequent $\mathrm{K}$-sets, the two algorithms are basically the same, and the time-modified Apriori algorithm for generating candidate frequent itemsets is obviously lower than Apriori algorithm, which shows that the improved algorithm significantly reduces the computing time, performance is higher than the Apriori algorithm.

Transform the Minimum Support. The minimal support min-sup is set to be $10 \%, 15 \%, 20 \%$, $25 \%, 30 \%$ respectively. Apriori and Apriori are used to generate candidate frequent $\mathrm{k}$-itemsets. To make the results more accurate, The algorithms are run three times to take the average time, using different support each operation time required as shown in Table 2 below:

Table 2. Transformation support two algorithms to generate frequent candidate K-term sets the timetable (ms)

\begin{tabular}{|c|c|c|c|c|c|}
\hline \multirow{2}{*}{ Support } & Algorithm & $\begin{array}{c}\text { First operation } \\
\text { time }\end{array}$ & $\begin{array}{c}\text { Second } \\
\text { operation time }\end{array}$ & $\begin{array}{c}\text { the third } \\
\text { operation time }\end{array}$ & $\begin{array}{c}\text { Average } \\
\text { operation time }\end{array}$ \\
\hline \multirow{2}{*}{$10 \%$} & Apriori & 3085 & 3127 & 3096 & 3098 \\
\cline { 2 - 6 } & Improved Apriori & 1172 & 1198 & 1205 & 1193 \\
\hline \multirow{2}{*}{$15 \%$} & Apriori & 1639 & 1708 & 1729 & 1696 \\
\cline { 2 - 6 } & Improved Apriori & 862 & 862 & 856 & 848 \\
\hline \multirow{2}{*}{$20 \%$} & Apriori & 918 & 923 & 939 & 928 \\
\cline { 2 - 6 } & Improved Apriori & 519 & 509 & 524 & 517 \\
\hline \multirow{2}{*}{$25 \%$} & Apriori & 759 & 747 & 765 & 759 \\
\cline { 2 - 6 } & Improved Apriori & 308 & 315 & 318 & 317 \\
\hline \multirow{2}{*}{$30 \%$} & Apriori & 428 & 437 & 419 & 428 \\
\cline { 2 - 6 } & Improved Apriori & 219 & 209 & 226 & 217 \\
\hline
\end{tabular}

It can be seen from the above figure that the algorithm of transformation support is used for testing. The improved Apriori algorithm has obvious advantages over Apriori algorithm. When the support degree is smaller, the performance of the improved algorithm is more prominent, and the advantage is more obvious. 


\section{Summary}

Based on the deep research of Apriori algorithm, this paper points out the limitations of Apriori algorithm and proposes a new improved algorithm. The improved algorithm only needs to scan the database once to achieve the same operation effect as the Apriori algorithm, and avoids the repeated judgment between frequent itemsets and directly obtains a higher candidate frequent itemset, thereby greatly improving the algorithm efficiency. Experimental results also show that the algorithm does improve the efficiency of Apriori algorithm. It is believed that this is of some significance to the problems of lack of information for solving the data explosion facing the present era.

\section{References}

[1] Ho Yue Shun. Research and Application of Association Rules Mining Technology [D]. Nanjing: Nanjing University of Aeronautics and Astronautics, 2010.

[2] Jiawei Han, Micheline Kamber. Data Mining Concepts and Techniques [M]. Beijing: Mechanical Industry Press, 2006.

[3] Lin Jiaxiong, yellow war. Improved Apriori Algorithm Based on Array Vector [J]. Computer Applications and Software, 2011,28 (5): 268-271.

[4] Peng L, Shizhao N, Zheng W, Ziwei J, Jianwu Y, Zhongxiang Q, Wangmo P. Predicting durations of online collective actions based on Peaks' heights [J]. Communications in Nonlinear Science and Numerical Simulation. 2018, 55: 338-354.

[5] Liu Yuwen. Research and improvement of Apriori algorithm based on cross linked list [J]. Computer Applications and Software, 2012,29 (5): 267-269.

[6] Zhang Chunsheng, Song Linlin. Segmentation support Apriori algorithm and its application [J]. Computer Engineering and Applications, 2010,46 (16): 157-159.

[7] Tan Pangning, Steinbach M, Kumar V. Introduction to Data Mining [M]. Beijing: Mechanical Industry Press, 2011. 